\title{
PRDM8 internal promoter hyperhydroxymethylation correlates with increased expression of the corresponding transcript in Down syndrome
}

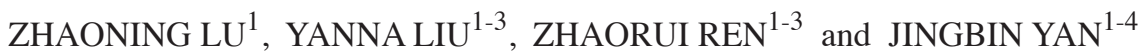 \\ ${ }^{1}$ Shanghai Institute of Medical Genetics; ${ }^{2}$ Shanghai Key Laboratory of Embryo and Reproduction Engineering, \\ Shanghai Children's Hospital, Shanghai Jiao Tong University, Shanghai 200040; \\ ${ }^{3}$ Key Laboratory of Embryo Molecular Biology, Ministry of Health of China, Shanghai 200040; \\ ${ }^{4}$ State Key Laboratory of Biotherapy, Collaborative Innovation Center for Biotherapy, \\ West China Hospital, Sichuan University, Chengdu, Sichuan 610041, P.R. China
}

Received May 22, 2015; Accepted February 24, 2016

DOI: $10.3892 / \mathrm{mmr} .2016 .5375$

\begin{abstract}
Down syndrome (DS) is the most common form of intellectual disability associated with central nervous system abnormalities and results from an extra complete or partial copy of human chromosome 21. However, whether DNA hydroxymethylation is perturbed in a specific gene associated with DS phenotypes, or the alteration of DNA hydroxymethylation results in changes of gene expression in DS remains unidentified. The current study mapped 5-methylcytosine and 5-hydroxymethylcytosine at $\mathrm{CpG}$ islands of the PR domain containing 8 (PRDM8) in the peripheral blood of 16 DS and 19 normal samples by oxidative bisulfite-pyrosequencing. Furthermore, the association of the expression levels of the two transcripts and epigenetic modification in different genomic contexts of PRDM8 was analyzed. The results demonstrated hypermethylation and hyperhydroxymethylation at the internal promoter of PRDM8 in DS, and significantly increased the expression of PRDM8 transcript variant 2 in the DS patients (median 3.9 vs. $2.04 ; \mathrm{P}=0.016$ ), accompanied by a positive correlation between the expression of two PRDM8 transcripts and hydroxymethylation at the corresponding external and internal promoters in patients, although not in the controls. A similar association was observed between the expression of transcript variant 1 and intragenic methylation of PRDM8.
\end{abstract}

Correspondence to: Dr Jingbin Yan, Shanghai Institute of Medical Genetics, Shanghai Children's Hospital, Shanghai Jiao Tong University, 24/1400 West Beijing Road, Shanghai 200040, P.R. China

E-mail: yanjingbin0130@hotmail.com

Abbreviations: OxBS-pyrosequencing, oxidative bisulfite pyrosequencing; BS-pyrosequencing, bisulfite pyrosequencing; CGI, CpG island; 5mC, 5-methylcytosine; $5 \mathrm{hmC}$, 5-hydroxymethylcytosine

Key words: PRDM8, DNA hydroxymethylation, DNA methylation, Down syndrome, transcriptional regulation
Taken together, the results of the present study suggest a critical role for DNA hydroxymethylation and methylation in regulating abnormal PRDM8 overexpression in DS.

\section{Introduction}

Down syndrome (DS) is the most common form of intellectual disability associated with central nervous system abnormalities and results from an extra complete or partial copy of human chromosome $21(1,2)$. It is likely that most DS phenotypes are associated with alterations in gene expression due to the supernumerary copy of chromosome 21 (3). Previous studies have demonstrated the upregulation of a certain subset of chromosome 21 genes, accompanied by numerous transcriptional changes throughout the genome $(3,4)$. Among the possible causes, alterations in epigenetic modifications may contribute to genome-wide changes in gene expression patterns in DS.

Epigenetics is the study of DNA methylation, patterns of histone modifications and non-coding RNAs that lead to changes in gene expression that are not accompanied by alterations in DNA sequence (5). Previous findings have shown that perturbation of DNA methylation is conserved in the peripheral blood leukocytes of adults with DS and in the placental villi of women with DS pregnancies. Differentially methylated genes have been identified on various autosomes in the leukocytes of patients with DS, and global DNA hypermethylation has been identified in the placenta of DS pregnancies $(6,7)$, suggesting that dysregulated methylation is an important cause of disrupted gene expression in DS. Furthermore, DNA methylation is perturbed to a greater extent in genes that are associated with DS phenotypes (7).

Using methylated DNA immunoprecipitation microarray (known as MeDIP-chip), 207 genes were identified with differential DNA methylation between the DS and normal controls, which may contribute to the clinical manifestations in DS (data not shown). For example, PR domain containing 8 (PRDM8), one of the genes hypermethylated in DS, participates in the development of the nervous system $(8,9)$. However, no correlation was identified between the extent of methylation 
and expression of certain differentially methylated genes in DS $(6,7)$. One explanation may be that DNA hydroxymethylation, an important regulator of gene expression, is involved but has gone undetected.

Five-hydroxymethylcytosine $(5 \mathrm{hmC})$ is an epigenetic DNA modification produced through the enzymatic activity of ten eleven translocation (TET) enzymes (10). TETs are 2-oxoglutarate- and $\mathrm{Fe}$ (II)-dependent dioxygenases that catalyze the hydroxylation of 5-methylcytosine $(5 \mathrm{mC})$ to $5 \mathrm{hmC}$ in the DNA (11). Further oxidation of $5 \mathrm{hmC}$ produces $5 \mathrm{fC}$ and 5-carboxylcytosine (5caC) (12). 5hmC acts as an intermediate involved in the DNA demethylation and as a functional epigenetic marker involved in gene regulation (13). $5 \mathrm{hmC}$ is highly enriched in the adult brain and accumulates across the lifespan and is markedly regulated by neural activity. Thus, $5 \mathrm{hmC}$ promotes rapid behavioral adaptation (14), suggesting that it has an important function in neural development. However, changes in hydroxylation of genes associated with the nervous system development have not been investigated in DS patients.

The aim of the present study was to investigate whether DNA hydroxymethylation is perturbed in a specific gene related to DS phenotypes and examine the functional relevancy of DNA hydroxymethylation to alterations of gene expression in DS.

\section{Materials and methods}

Samples from DS subjects and normal controls. Peripheral blood samples were obtained from 16 DS patients (age range, 2 days to 14 years) and 19 age-matched normal controls (age range, 13 days to 14 years). Informed consent was obtained from the parents of all the individuals. Mononuclear cells were freshly isolated using Lymphoprep (Axis-Shield Density Gradient Media; Alere Technologies AS, Oslo, Norway), and total RNA was extracted using TRIzol reagent (Ambion; Thermo Fisher Scientific, Inc., Waltham, MA, USA). RNA purity (A260/280 nm) was assessed using NanoDrop 2000 (Thermo Fisher Scientific, Inc.) and 2100 Bioanalyzer (Agilent Technologies, Inc., Santa Clara, CA, USA). DNA samples were extracted and purified using the Lab-Aid 820 Automated Blood DNA Extraction System (Xiamen Zeesan Biotech Co., Ltd., Fujian, China). The present study was approved by the Ethics Committee of Shanghai Children's Hospital, Shanghai Jiao Tong University (Shanghai, China).

Bisulfite pyrosequencing (BS-pyrosequencing). CGIs of PRDM8 were analyzed with MethPrimer (http://www.urogene.org/ cgi-bin/methprimer/methprimer.cgi), and $5 \mathrm{hmC}$ and $5 \mathrm{mC}$ levels were detected with BS pyrosequencing. BS-pyrosequencing was carried out as previously described (15). Briefly, genomic DNA (500 ng) from each sample was subjected to bisulfite treatment using the EpiTect Plus DNA Bisulfite kit (Qiagen GmbH, Hilden, Germany) according to the manufacturer's instructions. Pyrosequencing primers were designed using the PyroMark Assay Design software, version 2.0 (Qiagen $\mathrm{GmbH}$ ). Table I shows the primer sequences and length of PCR products. In each PCR reaction, one of the primers was labeled at the 5' flanking region with biotin (Invitrogen; Thermo Fisher Scientific. Inc.).

Bisulfite-treated DNA was amplified in the specific region using the PyroMark PCR kit (Qiagen $\mathrm{GmbH}$ ) according to the manufacturer's instructions. The PCR cycling conditions were as follows: $95^{\circ} \mathrm{C}$ for $15 \mathrm{~min}$, followed by 50 cycles of $30 \mathrm{sec}$ at $95^{\circ} \mathrm{C}, 30 \mathrm{sec}$ at $55^{\circ} \mathrm{C}$, and $30 \mathrm{sec}$ at $72^{\circ} \mathrm{C}$, with final extension at $72^{\circ} \mathrm{C}$ for $10 \mathrm{~min}$. The quality of the PCR products was evaluated by agarose gel electrophoresis (Biowest Agarose G-10; Gene Co., Ltd., Chai Wan, Hong Kong).

Templates were prepared using the PyroMark Q24 Vacuum Prep Workstation (Qiagen $\mathrm{GmbH}$ ) according to the manufacturer's instructions. Each single-stranded template was annealed to the sequencing primer $(0.375 \mu \mathrm{M})$ at $80^{\circ} \mathrm{C}$ for 5 min. Pyrosequencing was performed with the PyroMark Q24 system (Qiagen GmbH) using the PyroMark Q24 Advanced CpG Reagents (Qiagen $\mathrm{GmbH}$ ). The data were analyzed with the PyroMark Q24 Advanced (Qiagen GmbH).

Standard sample synthesis. To determine the conversion efficiency of $5 \mathrm{hmC}$ to uracil in oxidative bisulfite pyrosequencing (oxBS-pyrosequencing), a 49-nt standard sample containing three different cytosines $(\mathrm{C}, 5 \mathrm{mC}, 5 \mathrm{hmC})$ was designed and synthesized by GenScript (Nanjing City, China). The forward, reverse and sequencing standard sample primers were as follows: 5'-AGGAGGTTTAGAGTTTTGG-3' (F), 5'-ACCCAACTTACAAATTCTTTCTT-3' (RB) and 5'-AGGTTTAGAGTTTTGGT-3' (S), respectively (Invitrogen; Thermo Fisher Scientific. Inc.)

OxBS-pyrosequencing. The oxBS-pyrosequencing procedures were carried out as previously described $(16,17)$ with minor modifications. To optimize oxidation conditions, the 49-nt standard sample (50 ng) was denatured in $\mathrm{NaOH}(0.05 \mathrm{M})$ at $37^{\circ} \mathrm{C}$ for $30 \mathrm{~min}$ at room temperature. The solution was cooled at $4^{\circ} \mathrm{C}$ for $10 \mathrm{~min}$, followed by the oxidation reaction with $\mathrm{KRuO}_{4}$ (Sigma-Aldrich, St. Louis, MO, USA) at different concentrations $(0.6-2.5 \mathrm{mM})$ at $4^{\circ} \mathrm{C}$ for $1 \mathrm{~h}$. Each sample was then centrifuged at $14,000 \mathrm{x}$ g for $10 \mathrm{~min}$ to pellet any black precipitate. Subsequently, oxidized DNA was purified using the mini Quick Spin Oligo columns (Roche Diagnostics GmbH, Mannheim, Germany). BS-pyrosequencing was performed following the above protocol using the EpiTect Fast DNA Bisulfite kit (Qiagen GmbH).

To select the appropriate oxidation temperature, oxidation reactions were performed with $\mathrm{KRuO}_{4}(2 \mathrm{mM})$ at different temperatures $\left(4,16,25\right.$ and $\left.37^{\circ} \mathrm{C}\right)$ for $1 \mathrm{~h}$. The duration of bisulfite treatment was then optimized. Subsequent to the treatment of each 49-nt standard sample with $\mathrm{KRuO}_{4}(2 \mathrm{mM})$ at $4^{\circ} \mathrm{C}$ for $1 \mathrm{~h}, \mathrm{BS}$-pyrosequencing was carried out following the above bisulfite conversion with 1-4 amplification cycles.

To detect hydroxymethylation and methylation of PRDM8 in DS and normal control samples, oxBS-pyrosequencing was carried out following the above optimized protocol. In brief, genomic DNA $(500 \mathrm{ng})$ was incubated with $\mathrm{KRuO}_{4}(2 \mathrm{mM})$ for $1 \mathrm{~h}$ at $4^{\circ} \mathrm{C}$. Following purification of oxidized DNA using the mini Quick Spin Oligo column, oxidized and non-oxidized genomic DNA (500 ng) from the same sample were simultaneously subjected to 2 cycles of bisulfite conversion amplification using the EpiTect Plus DNA Bisulfite kit. The oxidized and non-oxidized DNA from each sample were then subjected to pyrosequencing.

Reverse transcription-quantitative polymerase chain reaction $(R T-q P C R)$. Reverse transcription was carried out with 
Table I. Primer sequences used for pyrosequencing.

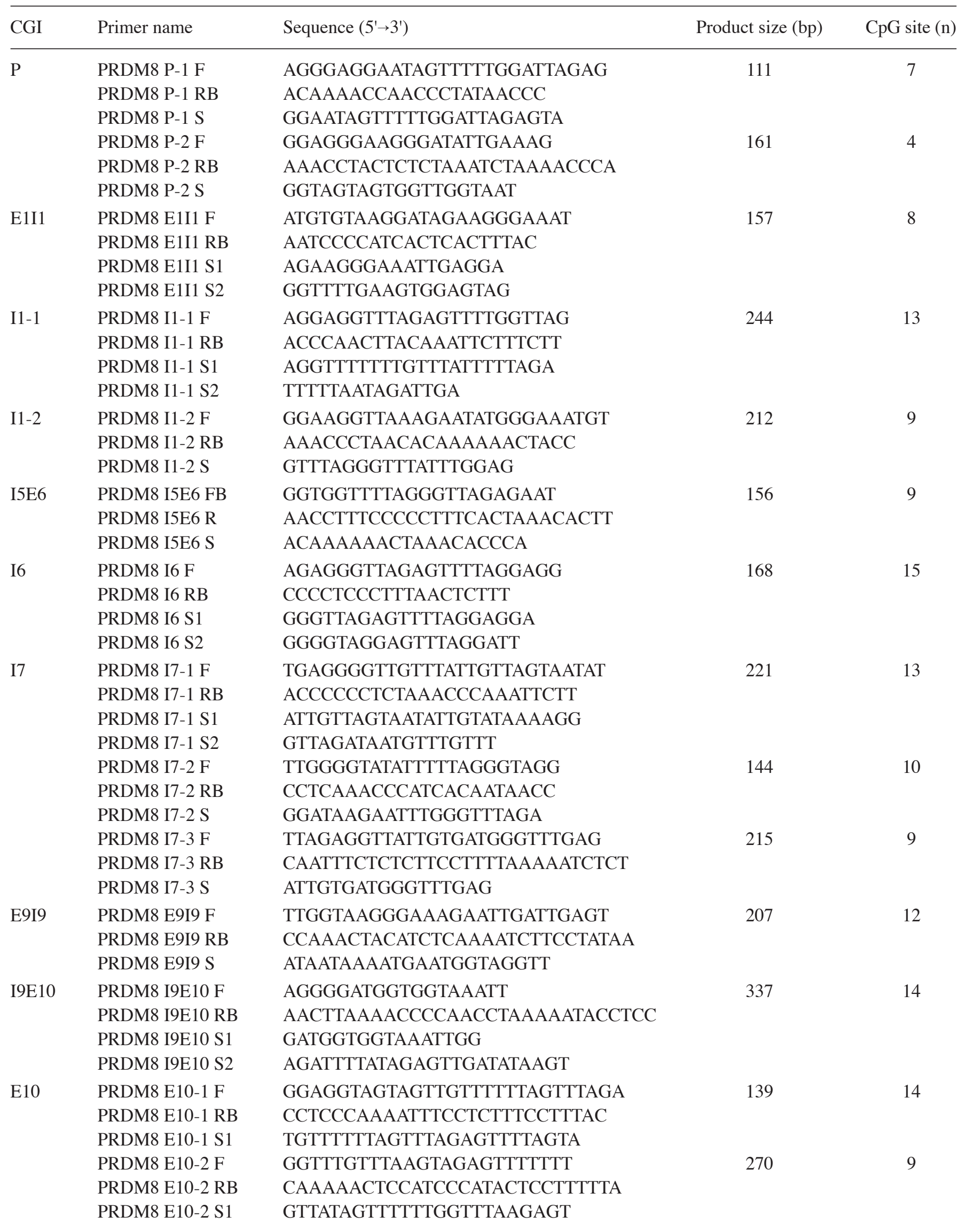

CGI, CpG island; bp, base pairs; PRDM8, PR domain containing 8; I5E6, represents the CGI across intron 5 and exon 6; I6, represents the CGI within intron 6; I7, represents the CGI within intron 7; E9I9, represents the CGI across exon 9 and intron 9; F, forward; R, reverse; FB, forward primer 5 ' biotin added; RB, reverse primer 5 ' biotin added; $S$, sequencing. 

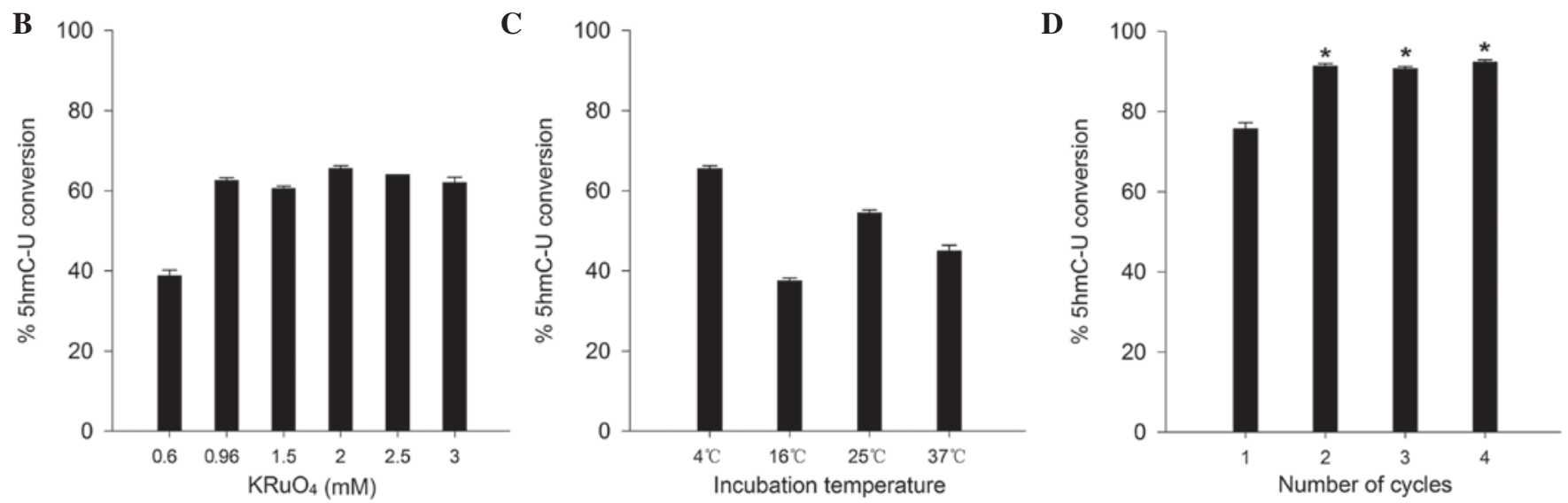

$\mathbf{E}$

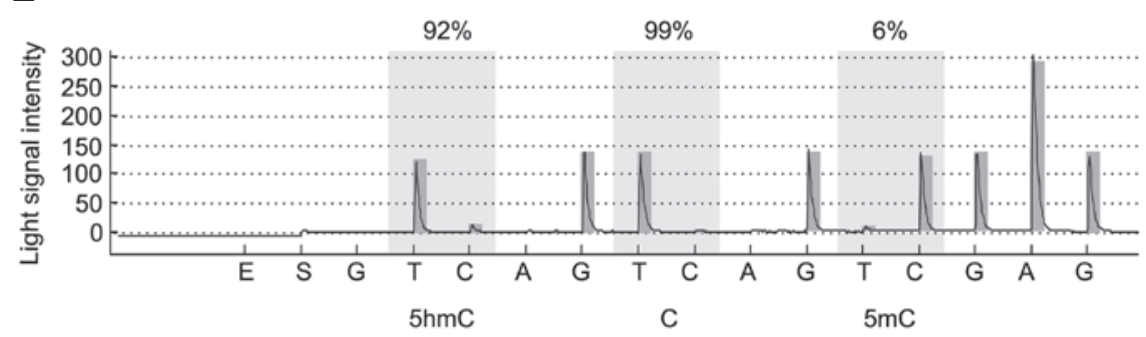

Figure 1. Conditions for oxBS. (A) A standard 49-residue oligonucleotide with three different modified cytosines: C, $5 \mathrm{mC}$, and $5 \mathrm{hmC}$. Conversion efficiency of $5 \mathrm{hmC}$ to uracil at $(\mathrm{B})$ different concentrations of the oxidant $\mathrm{KRuO}_{4}$ with constant oxidation temperature $\left(4^{\circ} \mathrm{C}\right)$ and $(\mathrm{C})$ different oxidation temperatures with a constant concentration of $\mathrm{KRuO}_{4}(2 \mathrm{mM})$. (D) Effect of the number of bisulfite conversion amplification cycles on the conversion efficiency of $5 \mathrm{hmC}$ to uracil with constant oxidation temperature $\left(4^{\circ} \mathrm{C}\right)$ and concentration of $\mathrm{KRuO}_{4}(2 \mathrm{mM}) .{ }^{*} \mathrm{P}<0.01$ vs. the first cycle. (E) OxBS-pyrosequencing results for a standard sample with the optimized protocol $\left(2 \mathrm{mM} \mathrm{KRuO}_{4}\right.$ treated at $4^{\circ} \mathrm{C}$ and 2 cycles of bisulfite conversion amplification using EpiTect Plus DNA Bisulfite kit). The three shaded areas show the ratio of $\mathrm{T}$ on three different sites $(5 \mathrm{hmC}, \mathrm{C}, 5 \mathrm{mC})$. Under these conditions, the highest conversion efficiency of $5 \mathrm{hmC}$ reached $92 \%$. 5 hmC-U, 5-hydroxymethylcytosine-Uracil; 5mC, 5-methylcytosine.

the RNase H Minus M-MLV reverse transcriptase (Takara Biotechnology Co., Ltd., Shiga, Japan) using $1 \mu \mathrm{g}$ total RNA and $0.5 \mu \mathrm{g}$ oligo (dT)18 primer (Shanghai Generay Biotech Co., Ltd., Shanghai, China). The reagent was incubated for $10 \mathrm{~min}$ at $70^{\circ} \mathrm{C}$, then placed in an ice bath for $2 \mathrm{~min}$. Subsequently, the reaction solution, including the M-MLV buffer, dNTPs, RNase inhibitor and M-MLV reverse transcriptase (Takara Biotechnology Co., Ltd.), was incubated at $42^{\circ} \mathrm{C}$ for $1 \mathrm{~h}$ and $70^{\circ} \mathrm{C}$ for $15 \mathrm{~min}$.

The relative expression of the two transcripts of PRDM8 was determined using the TaqMan Gene Expression Assay (Thermo Fisher Scientific, Inc.). Hs01027634_g1 was used for transcript variant 1 and a customized assay for variant 2, and Hs03929097_g1 was used for GAPDH as a control (Applied Biosystems; Thermo Fisher Scientific, Inc.). qPCR analysis was performed using $25 \mathrm{ng}$ cDNA and a QuantiNova Probe PCR Kit (Qiagen $\mathrm{GmbH}$ ), according to the manufacturer's protocol, on a 7500 system (Applied Biosystems; Thermo Fisher Scientific, Inc.) according to the manufacturer's protocol. The thermal profile for quantitative PCR was $95^{\circ} \mathrm{C}$ for $10 \mathrm{~min}$ followed by 40 cycles of $95^{\circ} \mathrm{C}$ for $15 \mathrm{sec}$ and $60^{\circ} \mathrm{C}$ for $60 \mathrm{sec}$. The relative expression levels were calculated using the $2^{-\Delta \Delta \mathrm{Cq}}$ normalization method and the values of PRDM8 and GAPDH (18).

Statistical analysis. Student's t-test (normally distributed data) and the Mann-Whitney test (non-normally distributed data) were used for comparing two groups. The Pearson's correlation was used for correlation analysis. Statistical analysis was performed using the SPSS 13.0 software (SPSS, Inc., Chicago, IL, USA). $\mathrm{P}<0.05$ was considered to indicate a statistically significant difference.

\section{Results}

Optimization of the oxBS-pyrosequencing protocol. To achieve maximal conversion efficiency of $5 \mathrm{hmC}$ to uracil $(5 \mathrm{hmC} \rightarrow 5 \mathrm{fC} \rightarrow \mathrm{U})$, the experimental conditions, including the appropriate concentration of oxidant $\left(\mathrm{KRuO}_{4}\right)$, oxidation temperature and time of bisulfite treatment, were optimized. A 49-nt standard sample containing three different cytosines (C, $5 \mathrm{mC}, 5 \mathrm{hmC}$ ) was designed and synthesized to determine the conversion efficiency of $5 \mathrm{hmC}$ to uracil in oxBS-pyrosequencing (Fig. 1A). The results indicated that the concentration of $\mathrm{KRuO}_{4}$ was an important factor affecting the conversion efficiency. As shown in Fig. 1B, the conversion efficiency improved from 38.7 to $62.5 \%$ following the change of concentration of $\mathrm{KRuO}_{4}$ from 0.6 to $0.96 \mathrm{mM}$. The highest efficiency of $5 \mathrm{hmC}$ conversion was $65.5 \%$ at $2 \mathrm{mM} \mathrm{KRuO}_{4}$, and thus this concentration was used in subsequent experiments (Fig. 1B).

To determine the appropriate oxidation temperature, standard samples were incubated with $\mathrm{KRuO}_{4}(2 \mathrm{mM})$ at different temperatures $\left(4,16,25\right.$ and $\left.37^{\circ} \mathrm{C}\right)$. The greatest conversion was achieved at $4^{\circ} \mathrm{C}$ (Fig. 1C), which was then used in subsequent 
A

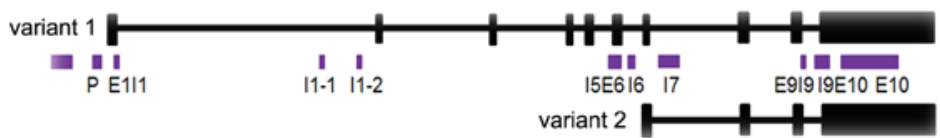

B

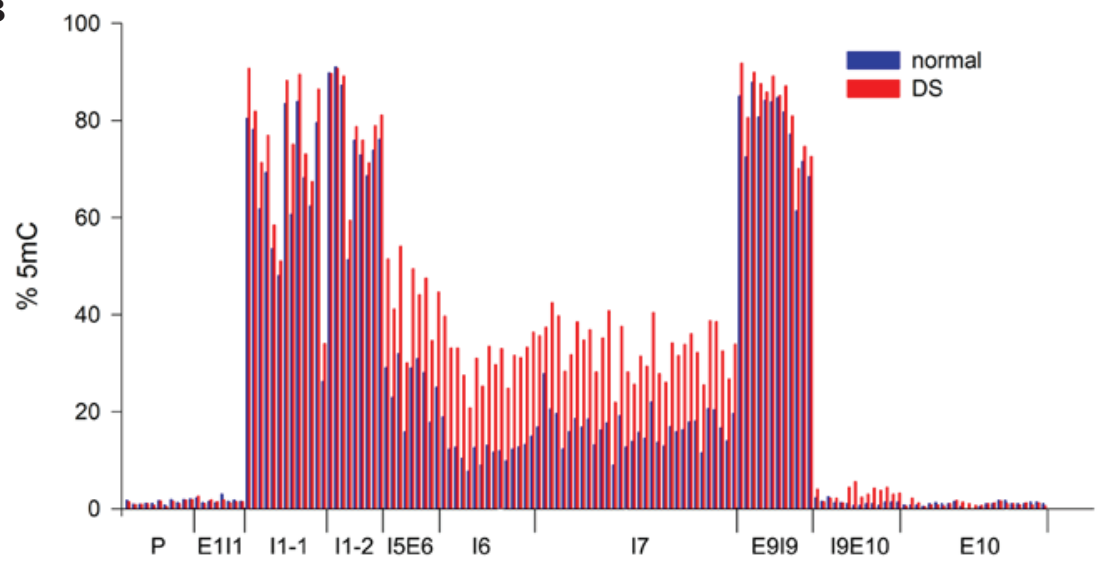

$\mathbf{C}$

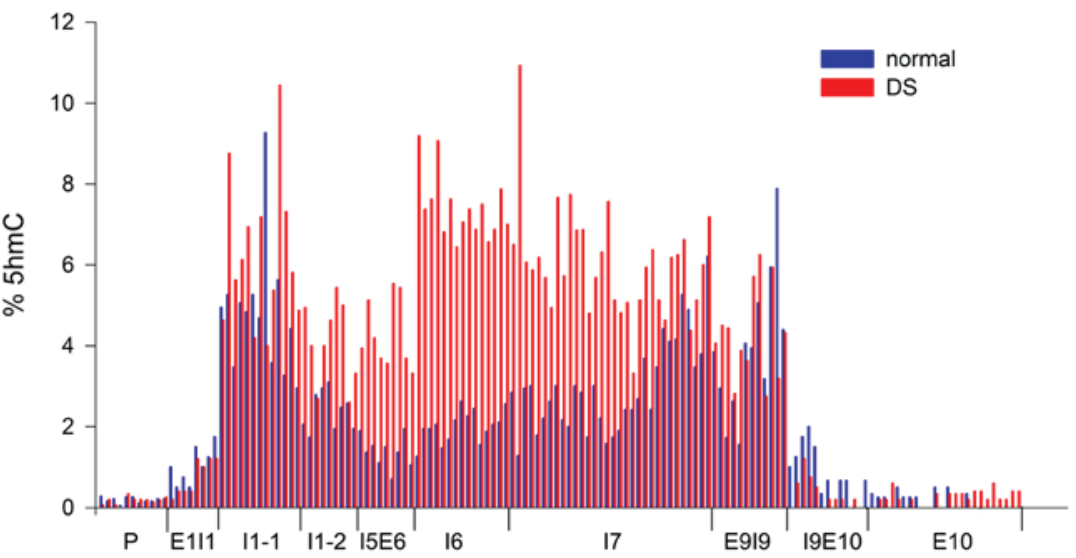

Figure 2. Levels of 5mC and 5hmC in PRDM8 from peripheral blood samples. (A) Sketch map of CGIs and two transcripts of PRDM8. CGIs of PRDM8 were analyzed with MethPrimer and are represented by the purple bars. Location of CGIs was denoted by P, E1I1, I1-1, I1-2, I5E6, I6, I7, E9I9, I9E10, E10, where $\mathrm{P}$ is the promoter, $\mathrm{E}$ is the exon and $\mathrm{I}$ is the intron. Black vertical bars represent exons of PRDM8. The long and short isoforms are transcript variants 1 and 2, respectively. (B) Averaged 5mC levels of PRDM8 in peripheral blood samples from children with DS and normal controls. The number of CGI samples sequenced was 16 from DS and 19 from normal samples for P, I1-1, I1-2, I5E6, I6, I7 and E9I9. The number sequenced for the remaining CGIs was 5 for DS and 4 for normal samples. (C) Averaged 5hmC levels of PRDM8 in peripheral blood samples from children with DS and normal controls. DS, Down syndrome; PRDM8, PR domain containing 8; 5hmC, 5-hydroxymethylcytosine; 5mC, 5-methylcytosine; CGI, CpG island.

experiments. Furthermore, to achieve a higher conversion efficiency, different numbers of bisulfite conversion amplification cycles (1-4 cycles) were attempted subsequent to the oxidation of the sample with $\mathrm{KRuO}_{4}(2 \mathrm{mM})$. The conversion efficiency of cycles 2, 3 and 4 was significantly increased, as compared with cycle 1 (Fig. 1D; $\mathrm{P}<0.01$ ); however, no significant differences were detected between cycles 2 , 3 and 4. Although the highest conversion efficiency was achieved with 4 cycles, the incorrect conversion of $5 \mathrm{mC}$ to uracil was elevated with the increasing cycle number (data not shown), and therefore 2 cycles were carried out in subsequent experiments. The optimized oxBS-pyrosequencing protocol is summarized as follows: the conversion efficiency of $5 \mathrm{hmC}$ to uracil reached $91.3 \%$ when the standard sample was treated with $2 \mathrm{mM} \mathrm{KRuO}_{4}$ at $4{ }^{\circ} \mathrm{C}$, followed by two cycles of bisulfite conversion amplification using the EpiTect Plus DNA Bisulfite kit (Fig. 1E).

Observation of hypermethylation and hyperhydroxymethylation in an internal promoter of PRDM8 in DS. Regions in PRDM8 containing different quantities of $5 \mathrm{mC}$ and $5 \mathrm{hmC}$ were identified with single-base resolution by oxBS-pyrosequencing of peripheral blood samples from children with DS and normal controls. Several CpG islands (CGIs) were detected in PRDM8 and two transcripts were encoded (Fig. 2A). The results indicated that $5 \mathrm{mC}$ and $5 \mathrm{hmC}$ were not evenly distributed across PRDM8, and the levels of methylation and hydroxymethylation were low in the 5' and 3' flanking regions (Fig. 2B and C). Hypermethylation was identified in 4 CGIs including I5E6 (represents the CGI across intron 5 and exon $6 ; 44.1$ vs. $25.6 \%$; $\mathrm{P}=5.63 \times 10^{-5}$ ), I6 (represents the CGI within intron $6 ; 30.9$ vs. $12.2 \%$; $\mathrm{P}=1.71 \times 10^{-4}$ ), I7 (represents the CGI within intron 7; 33.2 vs. $16.7 \%$ : $\mathrm{P}=3.49 \times 10^{-5}$ ), and E9I9 (represents the CGI across exon 9 and intron 9; 82.9 vs. $78.2 \%$; $\mathrm{P}=0.003$ ) in DS samples (Fig. 2B). The most significant hypermethylation was identified in the internal promoter region (I5E6, I6 and I7). Hyperhydroxymethylation was observed in only one CGI of patients DS (I6; 7.4 vs. 2.0\%; Fig. 2C; $\mathrm{P}=0.0046)$.

Expression level of PRDM8 transcript variant 2 is greater in DS. The expression levels of PRDM8 transcript variant 1 

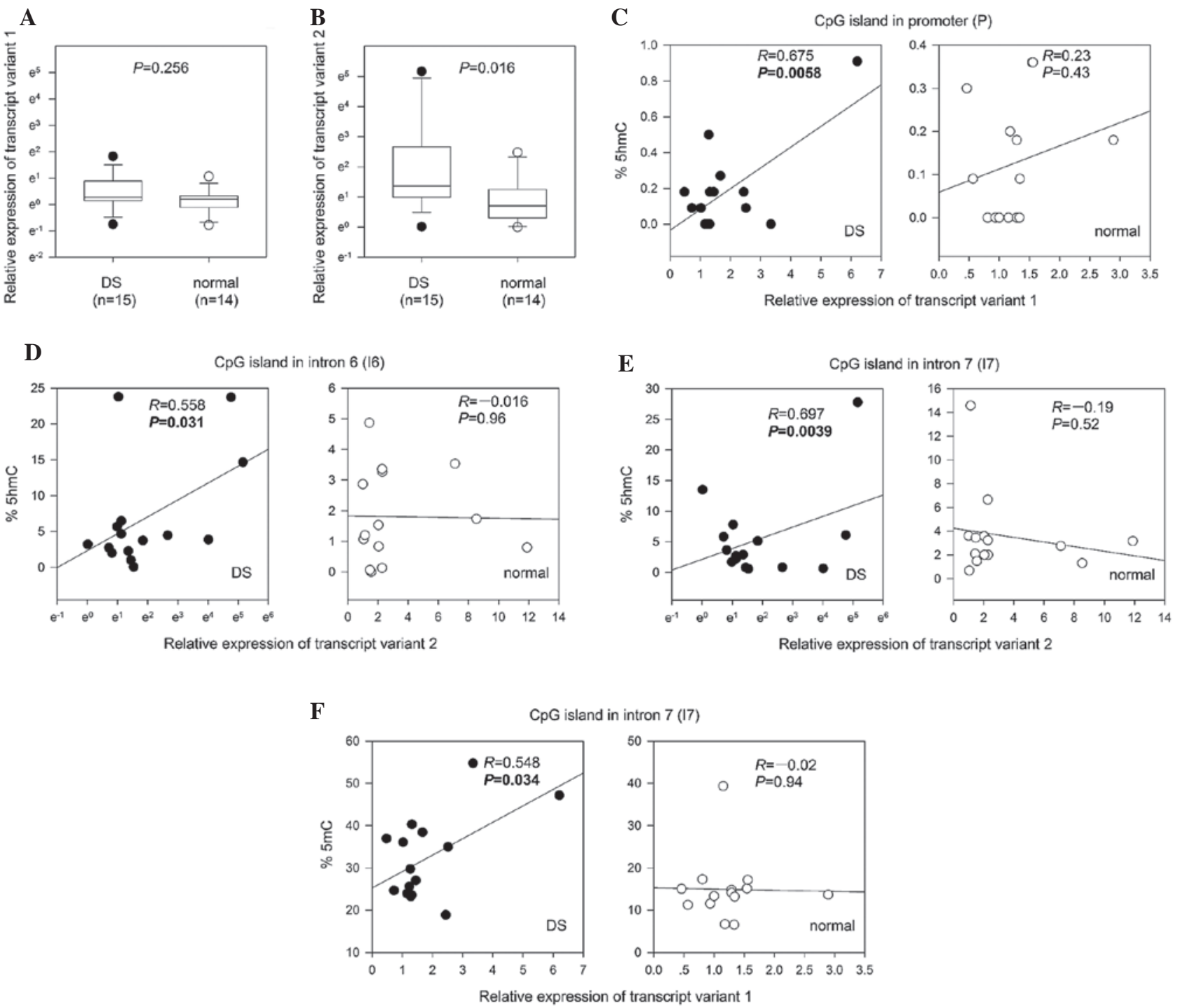

Figure 3. Levels of PRDM8 transcripts and their correlations with DNA hydroxymethylation/methylation. (A and B) Relative expression of PRDM8 transcript variants 1 and 2 in DS and normal samples. (C-F) Correlations between expression levels and 5hmC/5mC content at CGIs of different regions of PRDM8. The black and white circles represent DS and normal samples, respectively. DS, Down syndrome; PRDM8, PR domain containing 8; 5hmC, 5-hydroxymethylcytosine; 5mC, 5-methylcytosine; CGI, CpG island.

(long transcript) and transcript variant 2 (short transcript) were measured in 15 DS and 14 normal samples by qRT-PCR (Fig. 3A and B). The expression of transcript variant 2 was significantly higher in the DS group (median: 3.9 vs. 2.04; Fig. 3B; $\mathrm{P}=0.016)$. The level of transcript variant 1 was modestly higher in DS, but the difference was not statistically significant (median: 1.31 vs. 1.23; Fig. 3A; $\mathrm{P}=0.256$ ).

In DS, the expression levels of PRDM8 transcripts 1 and 2 were correlated with the external promoter and internal promoter hydroxymethylation, respectively (Figs 3C-E).

To investigate the regulation of PRDM8 expression in DS, the association of DNA methylation or hydroxymethylation of several CGIs (P, I1-1, I1-2, I5E6, I6, I7 and E9I9) in various genomic contexts (promoter and gene body), and the expression of the two transcripts was analyzed in DS and normal samples. As demonstrated in Fig. 3C, the expression of PRDM8 transcript variant 1 correlated positively with hydroxymethylation in the promoter region $(\mathrm{R}=0.675, \mathrm{P}=0.0058)$ in $\mathrm{DS}$, but not in the normal samples. Furthermore, as shown in Fig. 3D and E, a positive correlation was identified only in the DS samples among the expression of the PRDM 8 transcript variant 2 and hydroxymethylation of I6 $(\mathrm{R}=0.558, \mathrm{P}=0.0305)$ and $\mathrm{I} 7(\mathrm{R}=0.697$, $\mathrm{P}=0.0039$ ), located in the internal promoter region. These results suggested that hydroxymethylation in the promoter region is associated with higher levels of PRDM8 expression in DS.

Expression of PRDM8 transcript 1 correlates with internal promoter methylation in DS. The expression of PRDM8 transcript variant 1 correlated positively with methylation of the internal promoter region $(\mathrm{I} 7 ; \mathrm{R}=0.548, \mathrm{P}=0.034)$ in the $\mathrm{DS}$ samples, but not in the normal samples (Fig. 3F). These data suggested that intragenic methylation serves an important role in higher PRDM8 expression in DS.

\section{Discussion}

Changes in DNA methylation throughout the genome may be a reason for genome-wide alteration of gene expression in 
DS $(3,4)$, conserved in different tissues, particularly for genes associated with DS phenotypes $(6,7)$. Hydroxymethylation is a vital functional marker involved in neurogenesis. However, in previous studies, methods for mapping $5 \mathrm{mC}$ in DS have been based on bisulfite conversion of genomic DNA $(6,7)$, which cannot discriminate $5 \mathrm{mC}$ from $5 \mathrm{hmC}$. Thus, $5 \mathrm{hmC}$ is read as C following PCR amplification (19). Oxidative bisulfite sequencing (oxBS-Seq) is a novel method for quantitative mapping of $5 \mathrm{hmC}$ in genomic DNA at a single-nucleotide resolution (17). Selective chemical oxidation of $5 \mathrm{hmC}$ to 5 -formylcytosine (5fC) allows for bisulfite conversion of $5 \mathrm{fC}$ to uracil (17). Therefore, the difference between $5 \mathrm{hmC}$ and $5 \mathrm{mC}$ is be discriminated accurately through DNA sequencing. Due to the fundamental mechanism of oxBS-Seq, the approach is compatible with any sequencing platform. However, the reaction conditions of the oxidative bisulfite, that is important for accurate quantization, were not discussed in length in the previous studies. In the current study, pyrosequencing was used to detect the $5 \mathrm{mC}$ and $5 \mathrm{hmC}$ quantitatively through analyzing the ratio of $\mathrm{C} / \mathrm{T}$ in oxidized and non-oxidized genomic DNA from the same sample, and the oxBS-pyrosequencing protocol was investigated. The results demonstrate that the concentration of $\mathrm{KRuO}_{4}$ and the cycle of the bisulfite conversion may be two of the most important factors for improving conversion efficiency, as the conversion efficiency reached $91.3 \%$ following optimized conditions of oxBS-pyrosequencing.

PRDM8 is a key mediator of development, including the neurogenesis of the central nervous system $(8,9)$. PRDM8 has two alternative promoters that produce two transcripts, and several CGIs are located in different regions, including the promoter and gene body. In the current study, the expression of the PRDM8 transcript variant 2 was significantly higher in patients with DS. Therefore, PRDM8 is an optimal model gene for investigating the effects of epigenetic modifications on gene expression in DS.

Transcriptional regulation of DNA hydroxymethylation is associated with genomic contexts and cell types (20). Gene expression is generally suppressed by promoter hydroxymethylation in embryonic stem cells (ES cells) of humans (21) and mice $(22,23)$, whereas it is usually upregulated with gene body hydroxymethylation in mouse ES cells $(22,23)$ [but not in human ES cells (24)], neurons $(25,26)$, spermatogenic cells (27) and T cells (10). The results of the present study show that the expression of variants 1 and 2 correlates positively with the hydroxymethylation at the external and internal promoters, including I6 and I7, respectively, in the peripheral blood samples of patients with DS. This correlation was not apparent in the normal samples. A similar correlation has been identified in the human brain among the transcript levels and $5 \mathrm{hmC}$ content of promoters with low CpG content (28). These results suggest that DNA hyperhydroxymethylation may serve a critical role in the regulation of abnormal overexpression of PRDM8 transcript in DS.

It is well known that promoter methylation suppresses gene expression (29). The association of the intragenic methylation and gene expression is contradictory, varying with cell type and dependent on whether the methylated cytosine exists in a $\mathrm{CpG}$ or non-CpG context (20). Previous studies have suggested that the intragenic methylation contributes to higher gene expression in dividing cells, such as B lymphocytes, peripheral white blood cells, placenta and fibroblasts $(30,31)$. The results of the present study show that the expression of PRDM8 transcript variant 1 correlates positively with the intragenic methylation (I7) in DS, but not in normal samples, suggesting that intragenic methylation may be another mechanism for regulating PRDM8 expression in patients with DS.

In conclusion, the findings of the current study have demonstrated that alteration of hydroxymethylation and methylation of PRDM8 correlates with changes in its expression in the peripheral blood of children with DS. Given the proposed function of PRDM8 in cognitive disability in DS, we speculate that the alteration of epigenetic modification leading to abnormal PRDM8 expression may affect the transcription of a series of downstream genes that serve a critical role in abnormal central nervous system neurogenesis and development in patients with DS. Further studies of the epigenetic deregulation of PRDM8 using mouse models may aid to further elucidate the influence of PRDM8 upregulation on the nervous system development and to demonstrate the molecular mechanism(s) underlying DS in individual patients.

\section{Acknowledgements}

The present study was supported by the National Basic Research Project of China (grant nos. 2010CB529502 and 2007CB511904), the National Natural Science Foundation of China (grant no. 81471485), the key program for the fundamental research of the Science and Technology Commission of Shanghai (grant no. 11JC1411000), the Shanghai Joint Research Project for Important Disease (grant no. 2013ZYJB0015) and the Project from Shanghai Municipal Commission of Health and Family Planning (grant no. 20144Y0178).

\section{References}

1. Dierssen M: Down syndrome: The brain in trisomic mode. Nat Rev Neurosci 13: 844-858, 2012.

2. Haydar TF and Reeves RH: Trisomy 21 and early brain development. Trends Neurosci 35: 81-91, 2012.

3. Letourneau A, Santoni FA, Bonilla X, Sailani MR, Gonzalez D, Kind J, Chevalier C, Thurman R, Sandstrom RS, Hibaoui Y, et al: Domains of genome-wide gene expression dysregulation in Down's syndrome. Nature 508: 345-350, 2014.

4. Lockstone HE, Harris LW, Swatton JE, Wayland MT, Holland AJ and Bahn S: Gene expression profiling in the adult Down syndrome brain. Genomics 90: 647-660, 2007.

5. Rozek LS, Dolinoy DC, Sartor MA and Omenn GS: Epigenetics: Relevance and implications for public health. Annu Rev Public Health 35: 105-122, 2014.

6. Kerkel K, Schupf N, Hatta K, Pang D, Salas M, Kratz A, Minden M, Murty V, Zigman WB, Mayeux RP, et al: Altered DNA methylation in leukocytes with trisomy 21. PLoS Genet 6: e1001212, 2010.

7. Jin S, Lee YK, Lim YC, Zheng Z, Lin XM, Ng DP, Holbrook JD, Law HY, Kwek KY, Yeo GS, et al: Global DNA hypermethylation in down syndrome placenta. PLoS Genet 9: e1003515, 2013.

8. Inoue M, Kuroda T, Honda A, Komabayashi-Suzuki M, Komai T, Shinkai Y and Mizutani K: Prdm8 regulates the morphological transition at multipolar phase during neocortical development. PLoS One 9: e86356, 2014.

9. Ross SE, McCord AE, Jung C, Atan D, Mok SI, Hemberg M, Kim TK, Salogiannis J, Hu L, Cohen S, et al: Bhlhb5 and Prdm8 form a repressor complex involved in neuronal circuit assembly. Neuron 73: 292-303, 2012.

10. Tsagaratou A, Äijö T, Lio CW, Yue X, Huang Y, Jacobsen SE, Lähdesmäki $\mathrm{H}$ and Rao A: Dissecting the dynamic changes of 5-hydroxymethylcytosine in T-cell development and differentiation. Proc Natl Acad Sci USA 111: E3306-3315, 2014 
11. Tahiliani M, Koh KP, Shen Y, Pastor WA, Bandukwala H, Brudno Y, Agarwal S, Iyer LM, Liu DR, Aravind L, et al: Conversion of 5-methylcytosine to 5-hydroxymethylcytosine in mammalian DNA by MLL partner TET1. Science 324: 930-935, 2009.

12. Ito S, Shen L, Dai Q, Wu SC, Collins LB, Swenberg JA, He C and Zhang Y: Tet proteins can convert 5-methylcytosine to 5-formylcytosine and 5-carboxylcytosine. Science 333: 1300-1303, 2011.

13. $\mathrm{Wu} \mathrm{H}$ and Zhang Y: Reversing DNA methylation: Mechanisms, genomics, and biological functions. Cell 156: 45-68, 2014.

14. Li X, Wei W, Zhao QY, Widagdo J, Baker-Andresen D, Flavell CR, D'Alessio A, Zhang Y and Bredy TW: Neocortical Tet3-mediated accumulation of 5-hydroxymethylcytosine promotes rapid behavioral adaptation. Proc Natl Acad Sci USA 111: 7120-7125, 2014.

15. Mikeska T, Felsberg J, Hewitt CA and Dobrovic A: Analysing DNA methylation using bisulphite pyrosequencing. Methods Mol Biol 791: 33-53, 2011.

16. Booth MJ, Ost TW, Beraldi D, Bell NM, Branco MR, Reik W and Balasubramanian S: Oxidative bisulfite sequencing of 5-methylcytosine and 5-hydroxymethylcytosine. Nat Protoc 8: 1841-1851, 2013.

17. Booth MJ, Branco MR, Ficz G, Oxley D, Krueger F, Reik W and Balasubramanian S: Quantitative sequencing of 5-methylcytosine and 5-hydroxymethylcytosine at single-base resolution. Science 336: 934-937, 2012.

18. Livak KJ and Schmittgen TD: Analysis of relative gene expression data using real-time quantitative PCR and the 2(-Delta Delta C(T)) Method. Methods 25: 402-408, 2001.

19. Huang Y, Pastor WA, Shen Y, Tahiliani M, Liu DR and Rao A: The behaviour of 5-hydroxymethylcytosine in bisulfite sequencing. PLoS One 5: e8888, 2010.

20. Pastor WA, Aravind L and Rao A: TETonic shift: Biological roles of TET proteins in DNA demethylation and transcription. Nat Rev Mol Cell Biol 14: 341-356, 2013.

21. Stroud H, Feng S, Morey Kinney S, Pradhan S and Jacobsen SE: 5-Hydroxymethylcytosine is associated with enhancers and gene bodies in human embryonic stem cells. Genome Biol 12: R54, 2011
22. Huang Y, Chavez L, Chang X, Wang X, Pastor WA, Kang J, Zepeda-Martínez JA, Pape UJ, Jacobsen SE, Peters B, et al: Distinct roles of the methylcytosine oxidases Tet1 and Tet2 in mouse embryonic stem cells. Proc Natl Acad Sci USA 111: 1361-1366, 2014

23. Wu H, D'Alessio AC, Ito S, Wang Z, Cui K, Zhao K, Sun YE and Zhang Y: Genome-wide analysis of 5-hydroxymethylcytosine distribution reveals its dual function in transcriptional regulation in mouse embryonic stem cells. Genes Dev 25: 679-684, 2011

24. Szulwach KE, Li X, Li Y, Song CX, Han JW, Kim S, Namburi S, Hermetz K, Kim JJ, Rudd MK, et al: Integrating 5-hydroxymethylcytosine into the epigenomic landscape of human embryonic stem cells. PLoS Genet 7: e1002154, 2011.

25. Colquitt BM, Allen WE, Barnea G and Lomvardas S: Alteration of genic 5-hydroxymethylcytosine patterning in olfactory neurons correlates with changes in gene expression and cell identity. Proc Natl Acad Sci USA 110: 14682-14687, 2013.

26. Wen L, Li X, Yan L, Tan Y, Li R, Zhao Y, Wang Y, Xie J, Zhang Y, Song C, et al: Whole-genome analysis of 5-hydroxymethylcytosine and 5-methylcytosine at base resolution in the human brain. Genome Biol 15: R49, 2014.

27. Gan H, Wen L, Liao S, Lin X, Ma T, Liu J, Song CX, Wang M, He C, Han C, et al: Dynamics of 5-hydroxymethylcytosine during mouse spermatogenesis. Nat Commun 4: 1995, 2013.

28. Jin SG, Wu X, Li AX and Pfeifer GP: Genomic mapping of 5 -hydroxymethylcytosine in the human brain. Nucleic Acids Res 39: 5015-5024, 2011.

29. Jones PA: Functions of DNA methylation: Islands, start sites, gene bodies and beyond. Nat Rev Genet 13: 484-492, 2012.

30. Ball MP, Li JB, Gao Y, Lee JH, LeProust EM, Park IH, Xie B, Daley GQ and Church GM: Targeted and genome-scale strategies reveal gene-body methylation signatures in human cells. Nat Biotechnol 27: 361-368, 2009.

31. Aran D, Toperoff G, Rosenberg M and Hellman A: Replication timing-related and gene body-specific methylation of active human genes. Hum Mol Genet 20: 670-680, 2011. 